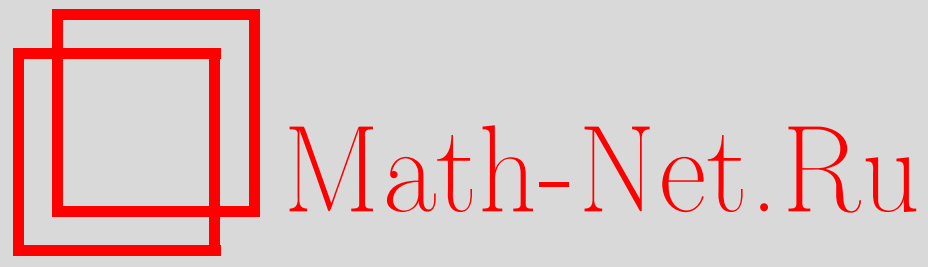

А. С. Шмелев, Симплектические связности на расслоениях регулярных орбит присоединенных представлений простых групп Ли, Функи. анализ и его прил., 1996, том 30, выпуск 1, 47-53

DOI: https://doi.org/10.4213/faa503

Использование Общероссийского математического портала MathNet.Ru подразумевает, что вы прочитали и согласны с пользовательским соглашением

http://www.mathnet.ru/rus/agreement

Параметры загрузки:

IP : 54.198 .64 .247

26 апреля 2023 г., 14:04:55

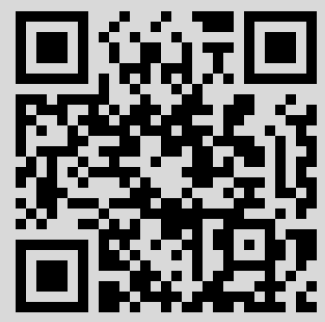


Функииональньй анализ и его приложения

1996, т. 30 , вып. 1 , с. $47-53$

УДК 515.164.15

\title{
Симплектические связности на расслоениях регулярных орбит присоединенных представлений простых групп Ли
}

\author{
(C) $1996 . \quad$ A. С. ШMелЕВ ${ }^{1}$
}

\section{§1. Введение}

Существуют интересные и неизученные связи между симплектической геометрией и теорией критических точек голоморфных функций. Естественным обобщением милноровского расслоения неособых поверхностей уровня функщии $f(\mathbf{z})=z_{1}^{2}+z_{2}^{2}+z_{3}^{2}$ являются расслоения регулярных орбит присоединенных представлений простых групп Ли (орбиту будем называть регулярной, если элементы этой орбиты не имеют совпадающих собственных чисел). Действительно, в случае $\mathrm{A}_{1} \cong \mathrm{C}_{1}$ (для группы $\mathrm{SL}(2, \mathbb{C})=\mathrm{Sp}(2, \mathbb{C})$ ) орбита однозначно определяется своим детерминантом $f(\xi)=\operatorname{det} \xi$. Если $\xi=\left(\begin{array}{cc}a & b \\ c & -a\end{array}\right)$, то в координатах $z_{1}=-i a, z_{2}=\frac{1}{2}(b-c), z_{3}=-\frac{i}{2}(b+c)$ функция $f$ принимает вид $f(\mathbf{z})=z_{1}^{2}+z_{2}^{2}+z_{3}^{2}$, и, следовательно, расслоение регулярных орбит $p: E \rightarrow B$ изоморфно милноровскому расслоению функции $f(\mathbf{z})=z_{1}^{2}+z_{2}^{2}+z_{3}^{2}$. В случае $\mathrm{B}_{1}=\mathrm{SO}(3, \mathbb{C})$ касательная алгебра состоит из матрищ

$$
\xi=\left(\begin{array}{ccc}
0 & a & b \\
-a & 0 & c \\
-b & -c & 0
\end{array}\right)
$$

с характеристическим полиномом $p(\xi)(\lambda)=-\lambda^{3}-\left(a^{2}+b^{2}+c^{2}\right) \lambda$. Здесь орбита определяется единственным инвариантом $f(a, b, c)=a^{2}+b^{2}+c^{2}$. Следовательно, и в этом случае расслоение регулярных орбит $p: E \rightarrow B$ изоморфно милноровскому расслоению функции $f(\mathbf{z})=z_{1}^{2}+z_{2}^{2}+z_{3}^{2}$.

Далее мы будем обозначать через $p: E \rightarrow B$ расслоения регулярных орбит присоединенных представлений классических простых групп Ли (типов $\mathrm{A}_{n}, \mathrm{~B}_{n}$, $\left.\mathrm{C}_{n}, \mathrm{D}_{n}\right)$, где $E$ есть объединение регулярных орбит. База $B$ и слой $F$ расслоения $p$ будут описаны ниже во всех четырех рассматриваемых случаях. Слой расслоения $p$ является симплектическим многообразием и диффеоморфен как вещественное многообразие касательному расслоению некоторого многообразия флагов $M$.

ОПРЕДЕлЕНИЕ 1. Связность на расслоении $p$ называется симплектической, если для любого пути в базе соответствующее отображение параллельного переноса является симплектоморфизмом.

${ }^{1}$ Настоящая работа выполнена при поддержке ISF, грант No. MSD000, и INTAS, грант No. 4373. 
Для каждого слоя $F=T_{*} M$ расслоения $p$ рассмотрим многообразие ориентированных касательных прямых к $M$, которое будем обозначать $S_{*} M$. Полученное расслоение $p_{\infty}: E_{\infty} \rightarrow B$, слой которого есть сферическое расслоение $S_{*} M$, может быть рассмотрено как бесконечно удаленное подрасслоение расслоения $p$. Произвольная связность не может быть продолжена на подрасслоение $p_{\infty}$, однако для связностей, имеющих такое продолжение, имеет смысл следующее определение.

ОПРЕДЕЛЕНИЕ 2. Связность на расслоении $p$ называется тождественной на бесконечности, если ее продолжение на расслоение $p_{\infty}$ является тождественной связностью (для любой петли в базе соответствующее отображение параллельного переноса является тождественным).

В работе [1] В. И. Арнольдом был приведен пример плоской симплектической связности, тождественной на бесконечности, на милноровском расслоении функции $f(\mathbf{z})=z_{1}^{2}+z_{2}^{2}+z_{3}^{2}$ и поставлен следующий вопрос. Можно ли выбрать гладко зависяшие от слоя симплектические структуры на слоях расслоения регулярных орбит присоединенного представления группы $\mathrm{SL}(n, \mathbb{C})$ так, чтобы на этом расслоении существовала плоская симплектическая связность, тождественная на бесконечности? В настоящей статье доказывается, что для простых групп Ли типов $\mathrm{A}_{n}, \mathrm{~B}_{n}, \mathrm{C}_{n}, \mathrm{D}_{n}$ при $n>1$ расслоение $p_{\infty}$ нетривиально и, следовательно, на соответствующем расслоении регулярных орбит не существует связности, тождественной на бесконечности, даже не обязательно плоской или симплектической. При $n=1$ во всех четырех случаях существует плоская симплектическая связность, тождественная на бесконечности. Кроме того, будет построена естественная плоская симплектическая связность для всех рассматриваемых типов групп Ли, не являющаяся тождественной на бесконечности.

\section{$\S 2$. Построение плоской симплектической связности на расслоении $p: E \rightarrow B$}

Вначале приведем описание базы и слоя расслоения $p$ для каждого из рассматриваемых типов групп $\left(\mathrm{A}_{n}, \mathrm{~B}_{n}, \mathrm{C}_{n}, \mathrm{D}_{n}\right)$ (см., например, [2]). Для каждой группы $G$ рассматриваемого типа обозначим через $G_{\mathbb{R}}$ соответствующую компактную вещественную форму, а через $W$ - группу Вейля системы корней.

1) Случай $\mathrm{A}_{n} . G=\mathrm{SL}(n+1, \mathbb{C}), G_{\mathbb{R}}=\mathrm{SU}(n+1), W=S_{n+1}$. Регулярные орбиты - это орбиты матрищ вида

$$
\Lambda=\left(\begin{array}{ccc}
\lambda_{1} & \ldots & 0 \\
\vdots & \ddots & \vdots \\
0 & \ldots & \lambda_{n+1}
\end{array}\right), \quad \lambda_{i} \in \mathbb{C}, \lambda_{i} \neq \lambda_{j} \text { при } i \neq j, \lambda_{1}+\cdots+\lambda_{n+1}=0 .
$$

Объединение $E$ регулярных орбит расслоено над пространством $B$ характеристических полиномов $t^{n+1}+a_{n-1} t^{n-1}+\cdots+a_{1} t+a_{0}$ без кратных корней, т. е. над пространством неупорядоченных наборов несовпадаюших комплексных чисел $\left(\lambda_{1}, \ldots, \lambda_{n+1}\right), \lambda_{1}+\cdots+\lambda_{n+1}=0$. Стабилизатор $G_{\Lambda} \subset \operatorname{SL}(n+1, \mathbb{C})$ элемента $\Lambda$ есть подгруппа диагональных матриц. Слой

$$
F=\mathrm{SL}(n+1, \mathbb{R}) / G_{\Lambda}=T_{*} \mathrm{SU}(n+1) / T_{*} \mathbb{T}^{n}=T_{*}\left(\mathrm{SU}(n+1) / \mathbb{T}^{n}\right)=T_{*} \mathbb{C F}_{n+1},
$$

где $\mathbb{C} F_{n+1}$ есть многообразие полных комплексных флагов в $\mathbb{C}^{n+1}$. 
2) Случай $\mathrm{B}_{n} \cdot G=\mathrm{SO}(2 n+1, \mathbb{C}), G_{\mathbb{R}}=\mathrm{SO}(2 n+1, \mathbb{R})$, а $W$ есть полупрямое произведение группы $(\mathbb{Z} / 2 \mathbb{Z})^{n}$ на $S_{n}$. Регулярные орбиты - это орбиты матриц вида

$$
\Lambda=\left(\begin{array}{cccc}
\Lambda_{1} & \ldots & 0 & 0 \\
\vdots & \ddots & \vdots & \vdots \\
0 & \ldots & \Lambda_{n} & 0 \\
0 & \ldots & 0 & 0
\end{array}\right), \quad \Lambda_{i}=\left(\begin{array}{cc}
0 & \lambda_{i} \\
-\lambda_{i} & 0
\end{array}\right), \Lambda_{i} \neq \pm \Lambda_{j}, \Lambda_{i} \neq 0, \lambda_{i} \in \mathbb{C} .
$$

Пространство $B$ есть факторпространство пространства упорядоченных наборов несовпадающих комплексных чисел $\left(\lambda_{1}, \ldots, \lambda_{n}\right), \lambda_{i} \neq 0$, по действию группы $W$, где первый сомножитель действует, меняя знаки, а второй - перестановками. Стабилизатор $G_{\Lambda}$ равен $\underbrace{\mathrm{SO}(2, \mathbb{C}) \times \cdots \times \mathrm{SO}(2, \mathbb{C})}_{n}$. Слой $F$ есть $T_{*} M$, где $M-$ многообразие ориентированных флагов в $\mathbb{R}^{2 n+1}$ вида

$$
\{0\} \subset \mathbb{R}^{1} \subset \mathbb{R}^{3} \subset \cdots \subset \mathbb{R}^{2 n-1} \subset \mathbb{R}^{2 n+1} .
$$

3) Случай $\mathrm{C}_{n} \cdot G=\operatorname{Sp}(2 n, \mathbb{C}), G_{\mathbb{R}}=\operatorname{Sp}(n)$ - унитарная кватернионная группа, а $W$ есть полупрямое произведение группы $(\mathbb{Z} / 2 \mathbb{Z})^{n}$ на $S_{n}$. Регулярные орбиты - это орбиты матриц вида

$$
\Lambda=\left(\begin{array}{ccc}
\Lambda_{1} & \ldots & 0 \\
\vdots & \ddots & \vdots \\
0 & \ldots & \Lambda_{n}
\end{array}\right), \quad \Lambda_{i}=\left(\begin{array}{cc}
\lambda_{i} & 0 \\
0 & -\lambda_{i}
\end{array}\right), \Lambda_{i} \neq \pm \Lambda_{j}, \Lambda_{i} \neq 0, \lambda_{i} \in \mathbb{C} .
$$

Пространство $B$ такое же, как в случае $\mathrm{B}_{n}$. Слой $F$ равен $T_{*} M$, где $M$ есть многообразие полных комплексных флагов в $\mathbb{C}^{2 n}=\mathbb{H}^{n}$, у которых четномерные подпространства являются кватернионными

$$
\{0\} \subset \mathbb{C}^{1} \subset \mathbb{H}^{1} \subset \mathbb{C}^{3} \subset \mathbb{H}^{2} \subset \cdots \subset \mathbb{C}^{2 n-1} \subset \mathbb{H}^{n} .
$$

4) Случай $\mathrm{D}_{n} . G=\mathrm{SO}(2 n, \mathbb{C}), G_{\mathbb{R}}=\mathrm{SO}(2 n, \mathbb{R})$, а $W$ есть полупрямое произведение $(\mathbb{Z} / 2 \mathbb{Z})^{n-1}$ на $S_{n}$. Регулярные орбиты - это орбиты матриц вида

$$
\Lambda=\left(\begin{array}{ccc}
\Lambda_{1} & \ldots & 0 \\
\vdots & \ddots & \vdots \\
0 & \ldots & \Lambda_{n}
\end{array}\right), \quad \Lambda_{i}=\left(\begin{array}{cc}
0 & \lambda_{i} \\
-\lambda_{i} & 0
\end{array}\right), \Lambda_{i} \neq \pm \Lambda_{j}, \Lambda_{i} \neq 0, \lambda_{i} \in \mathbb{C} .
$$

Пространство $B$ есть факторпространство пространства упорядоченных наборов несовпадающих комплексных чисел $\left(\lambda_{1}, \ldots, \lambda_{n}\right), \lambda_{i} \neq 0$, по действию группы $W$, где первый сомножитель действует, меняя знаки у четного числа элементов, а второй - перестановками. Слой $F$ равен $T_{*} M$, где $M$ есть многообразие ориентированных флагов в $\mathbb{R}^{2 n}$ вида

$$
\{0\} \subset \mathbb{R}^{2} \subset \mathbb{R}^{4} \subset \cdots \subset \mathbb{R}^{2 n-2} \subset \mathbb{R}^{2 n} .
$$

Перейдем к построению плоской симплектической связности на расслоении $p: E \rightarrow B$. Метрика Киллинга на алгебре Ли $\mathfrak{g}=T_{E} G$ индуцирует невырожденную метрику $\langle,\rangle_{\mathbb{C}}$ на пространстве расслоения $E \subset \mathfrak{g}$. Определим распределение $\mathscr{D}$ подпространств на $E$, ортогональных к слоям. Это распределение задает связность на расслоении $p$. 
ПРЕДЛОЖЕНИЕ 2.1. $\mathscr{D}$ есть плоская Аd-инвариантная связность с группой голономии $W$.

ДокаЗАтельство. Аd-инвариантность связности $\mathscr{D}$ вытекает из Ad-инвариантности метрики Киллинга. Пусть $N$ есть подмногообразие в $E$, состоящее из матриц вида (1) (соответственно $(2),(3),(4))$ в случае $\mathrm{A}_{n}$ (соответственно $\left.\mathrm{B}_{n}, \mathrm{C}_{n}, \mathrm{D}_{n}\right)$. Подмногообразие $N$ трансверсально пересекается с каждым слоем и касается распределения $\mathscr{D}$. Подмногообразия $\operatorname{Ad}_{A}(N)$ для всех $A \in G$ являются максимальными интегральными многообразиями, и, следовательно, распределение $\mathscr{D}$ вполне интегрируемо.

Теперь будем строить гладкое семейство симплектических форм на слоях расслоения $p: E \rightarrow B$ и плоскую симплектическую связность.

На слое $F$ действует группа голономии $W$ отображениями параллельного переноса связности $\mathscr{D}$. Пусть $P_{w}: F \rightarrow F$ есть отображение параллельного переноса, соответствующее элементу $w \in W$. Многообразие флагов $M$, соответствующее группе $G$, можно вложить в $F$ как орбиту присоединенного действия компактной подгруппы $G_{\mathbb{R}}$, выбирая любой элемент $\Lambda \in N \cap F$. Вложение $M \subset F$ не зависит от выбора $\Lambda$.

Лемма 2.2. Отображение $P_{w}: F \rightarrow F$ коммутирует с присоединенньм действием и сохраняет подмногообразие $M \subset F$ для любого $w \in W$.

Таким образом, можно определить подрасслоение $p_{\text {сотр }}: E_{\text {сотр }} \rightarrow B$ расслоения $p$, разнося подмногообразие $M$ отображениями параллельного переноса по всем слоям. Ограничение связности $\mathscr{D}$ на это подрасслоение дает связность на расслоении $p_{\text {comp }}$ с той же группой голономии $W$. Пусть $p_{w}: M \rightarrow M$ есть отображение параллельного переноса, соответствующее элементу $w \in W$. C расслоением $p_{\text {сотр }}: E_{\text {comp }} \rightarrow B$ связано расслоение $p_{1}: E_{1} \rightarrow B$, слои которого суть касательные расслоения слоев расслоения $p_{\text {сотр }}$. Расслоения $p$ и $p_{1}$ изоморфны. На расслоении $p_{1}: E_{1} \rightarrow B$ имеется плоская связность, определяемая отображениями параллельного переноса $p_{w^{*}}: T_{*} M \rightarrow T_{*} M, w \in W$.

Пусть $\langle$,$\rangle есть метрика на многообразии флагов M$, инвариантная относительно действия группы голономии $W$, и пусть $\omega=d p \wedge d q$ - лиувиллева форма на $T^{*} M$, а $\omega_{0}$ - симплектическая форма на $T_{*} M$, полученная из $\omega$ с помошью метрики $\langle$,$\rangle . Имеет место следующее утверждение.$

ЛЕмма 2.3. Форма $\omega_{0}$ инвариантна относительно изоморфизмов $f_{*}: T_{*} M \rightarrow T_{*} M$, индуцированных изометриями $f: M \rightarrow M$.

Таким образом, форма $\omega_{0}$ инвариантна относительно отображений параллельного переноса $p_{w^{*}}$ на расслоении $p_{1}: E_{1} \rightarrow B$ и однозначно разносится по всем слоям этого расслоения. Это дает плоскую симплектическую связность на расслоении $p: E \rightarrow B$, так как расслоения $p$ и $p_{1}$ изоморфны.

\section{$\S$ 3. Отсутствие связности, тождественной на бесконечности, на расслоении $p: E \rightarrow B$}

Будем рассматривать многообразие флагов $M$ как регулярную орбиту присоединенного представления соответствующей компактной вещественной формы $G_{\mathbb{R}}$. На алгебре Ли $\mathfrak{g}_{R}$ существует положительно определенное Ad-инвариантное скалярное произведение $\langle X, Y\rangle=\operatorname{Tr}\left(X \cdot Y^{*}\right)$, где $Y^{*}=\bar{Y}^{\mathrm{T}}$ в случаях $\mathrm{A}_{n}$ и $\mathrm{C}_{n}$ и $Y^{*}=Y^{\mathrm{T}}$ в случаях $\mathrm{B}_{n}$ и $\mathrm{D}_{n}$. Пусть $G_{X} \subset G_{\mathbb{R}}-$ стабилизатор некоторого элемента $X$ из орбиты $M$ и $\mathfrak{g}_{X}$ - его касательная алгебра. Можно считать, что $X \in N$. Имеет место следующее утверждение. 
ПРЕДЛОЖЕНИЕ 3.1 [3, §1]. Алгебра Ad-инвариантных дифференииальных форм на $M$ есть внешнял алгебра кососимметрических форм $\Lambda_{\text {inv }}^{*}\left(\mathfrak{g}_{\mathbb{R}} / \mathfrak{g}_{X}\right)$, обращающихся в нуль на $\mathfrak{g}_{X}$ и инвариантных относительно присоединенного действия стабилизатора $G_{X}$.

ЛЕмма 3.2. $\Lambda_{\text {inv }}^{1}\left(\mathfrak{g}_{\mathbb{R}} / \mathfrak{g}_{X}\right)=0$.

ДоказАтельство. Если $\alpha=\langle D, \cdot\rangle-$ некоторая форма из $\Lambda_{\text {inv }}^{1}\left(\mathfrak{g}_{\mathbb{R}} / \mathfrak{g}_{X}\right)$, то $\alpha\left(\operatorname{Ad}_{A} \xi\right)=\left\langle D, \operatorname{Ad}_{A} \xi\right\rangle=\left\langle\operatorname{Ad}_{A^{-1}} D, \xi\right\rangle=\alpha(\xi)=\langle D, \xi\rangle$ для любого $\xi \in \mathfrak{g}_{\mathbb{R}}$ и любого $A \in G_{X}$, т.е. $D \in \mathfrak{g}_{X} \cdot$ С другой стороны $\alpha(D)=\langle D, D\rangle=0$, т.е. $D=0$. Следовательно, $\Lambda_{\text {inv }}^{1}\left(\mathfrak{g}_{\mathbb{R}} / \mathfrak{g}_{X}\right)=0$.

Пусть $V$ - пространство кососимметрических 2-форм на алгебре Ли $\mathfrak{g}_{\mathbb{R}}$, имеющих вид $\omega_{D}(\xi, \eta)=\langle D,[\xi, \eta]\rangle, D \in \mathfrak{g}_{X}, V \subset \Lambda_{\text {inv }}^{2}\left(\mathfrak{g}_{\mathbb{R}} / \mathfrak{g}_{X}\right)$. По предложению 3.1 формы $\omega_{D}$ определяют Ad-инвариантные формы на $M$. Эти формы замкнуты и не когомологичны нулю; следовательно, $V$ отождествляется с подпространством в $H^{2}(M ; \mathbb{R})$.

Группа Вейля $W$ действует на пространстве $M$ отображениями параллельного переноса $p_{w}: M \rightarrow M$. По лемме 2.2 отображения $p_{w}$ коммутируют с присоединенным действием; следовательно, его дифференциал в точке $Y \in M$ имеет вид

$$
p_{w^{*}}[\xi, Y]=\left.\frac{d}{d t}\right|_{t=0} p_{w}\left(\operatorname{Ad}_{A(t)} Y\right)=\left.\frac{d}{d t}\right|_{t=0} \operatorname{Ad}_{A(t)}\left(p_{w}(Y)\right)=\left[\xi, p_{w}(Y)\right],
$$

где $\xi=\left.\frac{d}{d t}\right|_{t=0} \operatorname{Ad}_{A(t)} Y$.

Лемма 3.3. Группа Вейля $W$ действует на пространстве $V$ по формуле $p_{w}^{*} \omega_{D}(\nu, \mu)=\left\langle\operatorname{Ad}_{U} D,[\xi, \eta]\right\rangle$, где $\omega_{D}(\nu, \mu)=\langle D,[\xi, \eta]\rangle, \nu=[\xi, X], \mu=$ $[\eta, X], \nu, \mu \in T_{X} M$, a $U$ - такая матрича из $G_{\mathbb{R}}$, ито $\operatorname{Ad}_{U} X=p_{w}(X)$.

ДокАЗАТЕльство. В силу Аd-инвариантности формы $\omega_{D}$ имеем

$$
\begin{aligned}
\left(p_{w}^{*} \omega_{D}\right)(X)(\nu, \mu) & =\omega_{D}\left(p_{w}(X)\right)\left(\left[\xi, p_{w}(X)\right],\left[\eta, p_{w}(X)\right]\right) \\
& =\omega_{D}\left(\operatorname{Ad}_{U} X\right)\left(\left[\xi, p_{w}(X)\right],\left[\eta, p_{w}(X)\right]\right) \\
& =\operatorname{Ad}_{U}^{*}\left(\omega_{D}(X)\right)\left(\operatorname{Ad}_{U}^{-1}\left[\xi, p_{w}(X)\right], \operatorname{Ad}_{U}^{-1}\left[\eta, p_{w}(X)\right]\right) \\
& =\omega_{D}(X)\left(\left[\operatorname{Ad}_{U}^{-1} \xi, X\right],\left[\operatorname{Ad}_{U}^{-1} \eta, X\right]\right)=\left\langle D,\left[\operatorname{Ad}_{U}^{-1} \xi, \operatorname{Ad}_{U}^{-1} \eta\right]\right\rangle \\
& =\left\langle D, \operatorname{Ad}_{U}^{-1}[\xi, \eta]\right\rangle=\left\langle\operatorname{Ad}_{U} D,[\xi, \eta]\right\rangle . \quad \square
\end{aligned}
$$

СлЕдСТвИЕ 3.4. Представление $W \rightarrow \operatorname{Aut}(V)$ изоморфно представлению группы Вейля $W$, порожденному отражениями в следующих зеркалах:

1) Случай $\mathrm{A}_{n} \cdot V=\left\{x_{1}+\cdots+x_{n+1}=0\right\} \subset \mathbb{R}^{n+1}$.

Система зеркал: $\left\{x_{i}=x_{j}, i<j\right\}$.

2) Cлучаи $\mathrm{B}_{n}$ u $\mathrm{C}_{n} \cdot V=\mathbb{R}^{n}$.

Система зеркал: $\left\{x_{i}=x_{j}, i<j\right\}$ u $\left\{x_{i}=0, i=1, \ldots, n\right\}$.

3) Cлучай $\mathrm{D}_{n} \cdot V=\mathbb{R}^{n}$.

Система зеркал: $\left\{x_{i}=x_{j}, i<j\right\}$ u $\left\{x_{i}=-x_{j}, i<j\right\}$.

Теперь покажем, что во всех случаях, кроме $\mathrm{A}_{1} \cong \mathrm{B}_{1} \cong \mathrm{C}_{1}$ и $\mathrm{D}_{1}$, на расслоении $p: E \rightarrow B$ не существует связности, тождественной на бесконечности, или, иными словами, что бесконечно удаленное расслоение $p_{\infty}: E_{\infty} \rightarrow B$ нетривиально. (В случае $\mathrm{D}_{1}$ расслоение $p: E \rightarrow B$ тривиально, так как $p-$ диффеоморфизм.) 
Построенная выше связность на расслоении $p: E \rightarrow B$ продолжается на бесконечность. Обозначим через $S_{*} p_{w}: S_{*} M \rightarrow S_{*} M$ отображение параллельного переноса в этой связности, соответствуюшее элементу $w \in W$. Рассмотрим представление $\beta: \pi_{1}(B) \rightarrow W \rightarrow \operatorname{Aut}(V)$ фундаментальной группы базы в группу автоморфизмов пространства $V$.

Теорема 3.5. Расслоение $p_{\infty}: E_{\infty} \rightarrow B$ нетривиально во всех случаях, кроме $\mathrm{A}_{1} \cong \mathrm{B}_{1} \cong \mathrm{C}_{1}$.

ДокаЗАТЕЛЬСтво. Докажем, что построенное представление $\beta: \pi_{1}(B) \rightarrow$ Aut $(V)$ нетривиально, т. е. что его образ отличен от тождественного преобразования.

Пусть $d=\operatorname{dim}_{\mathbb{R}} M$, и пусть $\pi: S_{*} M \rightarrow M$ есть расслоение со слоем $S^{d-1} \subset$ $T_{f} M$. Для каждой петли в базе $B$ соответствующее ей отображение параллельного переноса $p_{w}: M \rightarrow M$ может либо сохранять ориентацию на $M$, либо менять ее на противоположную. Пусть $u \in H^{d-1}\left(S_{*} M ; \mathbb{R}\right)$ - некоторая ориентация на ориентируемом расслоении $\pi: S_{*} M \rightarrow M$ (рассматриваются когомологии де Рама). Тогда $\left(S_{*} p_{w}\right)^{*} u= \pm u=u^{\prime}$. Рассмотрим точные последовательности Гизина для этих двух ориентаций

$$
\begin{gathered}
\cdots \rightarrow H^{i}(M) \stackrel{\wedge e}{\longrightarrow} H^{i+d}(M) \stackrel{\pi^{*}}{\rightarrow} H^{i+d}\left(S_{*} M\right) \stackrel{p}{\rightarrow} H^{i+1}(M) \rightarrow \cdots, \\
\cdots \rightarrow H^{i}(M) \stackrel{\wedge e^{\prime}}{\longrightarrow} H^{i+d}(M) \stackrel{\pi^{*}}{\longrightarrow} H^{i+d}\left(S_{*} M\right) \stackrel{p^{\prime}}{\rightarrow} H^{i+1}(M) \rightarrow \cdots,
\end{gathered}
$$

где $e$ и $e^{\prime}= \pm e$ суть классы Эйлера, соответствующие ориентациям $u$ и $u^{\prime}$, а $p$ и $p^{\prime}= \pm p$ - гомоморфизмы интегрирования вдоль слоев. В силу функториальных свойств последовательности Гизина имеет место следующая коммутативная диаграмма:

$$
\begin{aligned}
& \cdots \stackrel{\wedge e^{\prime}}{\longrightarrow} H^{i+d}(M) \stackrel{\pi^{*}}{\longrightarrow} H^{i+d}\left(S_{*} M\right) \stackrel{p^{\prime}}{\longrightarrow} H^{i+1}(M) \longrightarrow \cdots \\
& \uparrow p_{w}^{*} \quad \uparrow\left(S_{*} p_{w}\right)^{*} \quad \uparrow p_{w}^{*} \\
& \cdots \stackrel{\wedge e}{\longrightarrow} H^{i+d}(M) \stackrel{\pi^{*}}{\longrightarrow} H^{i+d}\left(S_{*} M\right) \stackrel{p}{\longrightarrow} H^{i+1}(M) \longrightarrow \cdots
\end{aligned}
$$

Так как $H^{i+d}(M)=0$ при $i>0$, получаем следующую коммутативную диаграмму:

$$
\begin{aligned}
& 0 \longrightarrow H^{d+1}\left(S_{*} M\right) \stackrel{ \pm p}{\longrightarrow} H^{2}(M) \longrightarrow 0 \\
& \uparrow\left(S_{*} p_{w}\right)^{*} \quad \uparrow p_{w}^{*} \\
& 0 \longrightarrow H^{d+1}\left(S_{*} M\right) \stackrel{p}{\longrightarrow} H^{2}(M) \longrightarrow 0
\end{aligned}
$$

где вертикальные стрелки - изоморфизмы, а горизонтальные строки точные. Следовательно, представление

$$
\pi_{1}(B) \rightarrow \operatorname{Aut}\left(H^{*}\left(S_{*} M ; \mathbb{R}\right)\right)
$$

нетривиально в случаях, отличных от $\mathrm{A}_{1} \cong \mathrm{B}_{1} \cong \mathrm{C}_{1}$, так как представление $\pi_{1}(B) \rightarrow \operatorname{Aut}(V) \subset \operatorname{Aut}\left(H^{2}(M ; \mathbb{R})\right)$ нетривиально.

Таким образом, во всех случаях $\mathrm{A}_{n}, \mathrm{~B}_{n}, \mathrm{C}_{n}, \mathrm{D}_{n}, n>1$, на расслоении $p: E \rightarrow B$ регулярных орбит присоединенного представления не существует 
связности, тождественной на бесконечности. В случае $\mathrm{A}_{1} \cong \mathrm{B}_{1} \cong \mathrm{C}_{1}$ такая связность существует. В [1] приведен пример плоской симплектической связности, тождественной на бесконечности. Этот пример можно обобшить на случай милноровского расслоения функции $f(\mathbf{z})=z_{1}^{2}+\cdots+z_{n}^{2}$, имеющей особенность типа $\mathrm{A}_{1}$. В этом случае имеем расслоение $p: E \rightarrow \mathbb{C} \backslash\{0\}$ со слоем $F_{z}=p^{-1}(z)$. Поскольку база этого расслоения стягивается на окружность $S^{1}=$ $\left\{e^{i t}: t \in \mathbb{R}\right\}$, достаточно построить связность на ограничении расслоения на единичную окружность $p: E_{1} \rightarrow S^{1}$. Слой $F_{z}$ вещественно диффеоморфен кокасательному расслоению $T^{*} S^{n-1}$.

Вначале построим связность как семейство диффеоморфизмов $f_{t}: F_{1} \rightarrow F_{z}$, $z=e^{i t}$, заданных по формуле

$$
f_{t}\left(z_{1}, \ldots, z_{n}\right)=e^{i t / 2}\left(z_{1}, \ldots, z_{n}\right) .
$$

После выбора некоторого диффеоморфизма $h: F_{1} \rightarrow T^{*} S^{n-1}$ отображение $f_{2 \pi}: F_{1} \rightarrow F_{1}$ переходит в антиподальное $i^{*}: T^{*} S^{n-1} \rightarrow T^{*} S^{n-1}$. Пусть $(x, y)$ - координаты Дарбу на слое $F_{1}=T^{*} S^{n-1}$ и $\omega=d x \wedge d y$ - лиувиллева форма. Поскольку $i^{*}$ - симплектоморфизм, эта форма однозначно разносится по всем слоям расслоения, превращая построенную связность в симплектическую. Однако эта связность не тождественна на бесконечности.

Для построения тождественной на бесконечности связности рассмотрим гамильтоново векторное поле $\mathbf{v}$ на слое $F_{1}$ с гамильтонианом

$$
H(x, y)=\frac{2}{\pi}\|x\| \operatorname{arctg}\|x\| \text {. }
$$

Пусть $\left\{g_{t}\right\}$ - соответствующий гамильтонов поток. Отображение $g_{\pi}$ тождественно на нулевом сечении, и для продолжений антиподального изоморфизма $i^{*}$ и отображения $g_{\pi}$ на бесконечность $S^{*} S^{n-1}$ имеет место тождество $g_{\pi}=i^{*}$ на $S^{*} S^{n-1}$. Следовательно, отображение $i^{*} \circ g_{\pi}$ сохраняет симплектическую форму $\omega$ на $F_{1}$, тождественно на бесконечности и антиподально на нулевом сечении, т. е. является многомерным симплектическим обобщением скручивания Дена на двумерном цилиндре [4, введение].

Теперь определим связность на расслоении $p: E_{1} \rightarrow S^{1}$ семейством диффеоморфизмов $f_{2 t} \circ g_{t}: F_{1} \rightarrow F_{z}, z=e^{i t}, t \in \mathbb{R}$. Тогда отображение параллельного переноса, соответствующее полному обходу по окружности, совпадает с $i^{*} \circ g_{\pi}$. Это дает плоскую симплектическую тождественную на бесконечности связность на милноровском расслоении функции $f(\mathbf{z})=z_{1}^{2}+\cdots+z_{n}^{2}$.

\section{ЛИТЕРАТУРА}

1. Arnold $V$. Symplectic monodromy of Milnor fibrations. A Floer memorial volume (E. Zehnder ed.), Vol. 3, Birkhauser (1992).

2. Бурбаки H. Группы и алгебры Ли. Мир, М. (1972).

3. Дубровин Б. А., Новиков С. П., Фоменко А. Т. Современная геометрия. Методы теории гомологий. Наука, М. (1984).

4. Арнольд В.И., Варченко А. Н., Гусейн-Заде С. М. Особенности дифференцируемых отображений. II. Наука, М. (1984). 